\title{
LEVEL SET DYNAMICS AND THE NON-BLOWUP OF THE 2D QUASI-GEOSTROPHIC EQUATION*
}

\author{
J. DENG ${ }^{\dagger}$, T. Y. HOU ${ }^{\ddagger}$, R. $\mathrm{LI}^{\S}$, AND X. YU
}

\begin{abstract}
In this article we apply the technique proposed in Deng-Hou-Yu [7] to study the level set dynamics of the 2D quasi-geostrophic equation. Under certain assumptions on the local geometric regularity of the level sets of $\theta$, we obtain global regularity results with improved growth estimate on $\left|\nabla^{\perp} \theta\right|$. We further perform numerical simulations to study the local geometric properties of the level sets near the region of maximum $\left|\nabla^{\perp} \theta\right|$. The numerical results indicate that the assumptions on the local geometric regularity of the level sets of $\theta$ in our theorems are satisfied. Therefore these theorems provide a good explanation of the double exponential growth of $\left|\nabla^{\perp} \theta\right|$ observed in this and past numerical simulations.
\end{abstract}

Key words. Quasi-geostrophic equation, finite time blow-up, geometric properties, global existence

AMS subject classifications. Primary 76B03; Secondary 35L60, 35M10

1. Introduction. The study of global existence/finite-time blow-up of the twodimensional quasi-geostrophic (subsequently referred to as 2D QG for simplicity) equation has been an active research area in the past ten years, partly due to its close connection to the 3D incompressible Euler equations (Constantin-Majda-Tabak [2], Cordoba [5], Cordoba-Fefferman [6]). The 2D QG equation has its origin in modeling rotating fluids on the earth surface (Pedlosky [10]). The equation describes the transportation of a scalar quantity $\theta$ :

$$
D_{t} \theta \equiv \theta_{t}+u \cdot \nabla \theta=0
$$

with initial conditions $\left.\theta\right|_{t=0}=\theta_{0}$. The relation between $\theta$ and the velocity $u$ is given by

$$
u=\nabla^{\perp} \psi, \quad \psi=(-\triangle)^{-\frac{1}{2}}(-\theta)
$$

where

$$
\nabla^{\perp} \psi \equiv\left(-\frac{\partial \psi}{\partial x_{2}}, \frac{\partial \psi}{\partial x_{1}}\right)^{T}
$$

and

$$
(-\triangle)^{-\frac{1}{2}} \psi \equiv \int e^{2 \pi i x \cdot k} \frac{1}{2 \pi|k|} \hat{\psi}(k) \mathrm{d} k
$$

where $\hat{\psi}(k)=\int e^{-2 \pi i x \cdot k} \psi(x) \mathrm{d} x$ is the Fourier transform of $\psi(x)$.

\footnotetext{
${ }^{*}$ Received January 18, 2006; accepted for publication July 18, 2006.

†School of Mathematical Sciences, Fudan University, Shanghai, China (jdeng@fudan.edu.cn).

¥Applied and Computational Mathematics, California Institute of Technology, Pasadena, CA 91125, USA (hou@acm.caltech.edu).

$\S$ Applied and Computational Mathematics, California Institute of Technology, Pasadena, CA 91125, USA (rli@acm.caltech.edu).

『Department of Mathematics, University of California, Los Angeles, CA 90095, USA (xinweiyu @math.ucla.edu).
} 
As pointed out by Constantin-Majda-Tabak [2], the 2D QG equation bears striking mathematical and physical analogy to the 3D incompressible Euler equations. They both exhibit similar geometric/analytic structures. In particular, one can derive a necessary and sufficient blow-up condition for the 2D QG equation similar to the well-known Beale-Kato-Majda criterion (Beale-Kato-Majda [1]). More precisely, the solution to the $2 \mathrm{D}$ QG equation (1) becomes singular at time $T_{*}$ if and only if

$$
\int_{0}^{T_{*}}\left\|\nabla^{\perp} \theta(\cdot, t)\right\|_{L^{\infty}} \mathrm{d} t=+\infty
$$

Thus, $\nabla^{\perp} \theta$ plays a role similar to the vorticity $\omega$ in the 3D Euler equations. Furthermore, as in the 3D incompressible Euler equations, the velocity $u$ is related to $\nabla^{\perp} \theta$ by an order -1 singular integral operator.

On the other hand, in some aspects the 2D QG equation behaves much better than the 3D incompressible Euler equations. For example, it was shown in Cordoba [5] that $\|u(\cdot, t)\|_{L^{\infty}}$ is bounded by $\log \left\|\nabla^{\perp} \theta(\cdot, t)\right\|_{L^{\infty}}$ for any time $t$, while for the 3D incompressible Euler equations, $\|u(\cdot, t)\|_{L^{\infty}}$ may grow as fast as $\|\omega(\cdot, t)\|_{L^{\infty}}^{1 / 2}$ according to Kelvin's circulation theorem.

Much effort has been made to obtain global existence for the 2D QG equation. In Constantin-Majda-Tabak [2], it was shown that if the direction field $\xi \equiv \nabla^{\perp} \theta /\left|\nabla^{\perp} \theta\right|$ remains smooth in a region, then no finite-time singularity is possible in that region. In particular, if this region contains maximum $\left|\nabla^{\perp} \theta\right|$ all the time, then the solution remains regular global in time. Based on this understanding, they conjectured that the 2D QG equation with initial level sets of the hyperbolic saddle type is likely to develop a finite time singularity. They further presented some numerical evidence which supports a finite time singularity for the 2D QG equation. Later on, OhkitaniYamada [9], Constantin-Nie-Schorghofer [3], [4] re-did the numerical simulations with higher resolutions, and revealed that the growth of $\left\|\nabla^{\perp} \theta\right\|_{L^{\infty}}$ in time is no faster than double exponential. Around the same time, Cordoba [5] proved that the growth of $\left\|\nabla^{\perp} \theta\right\|_{L^{\infty}}$ in the hyperbolic saddle scenario is bounded by quadruple exponential under the assumption that near the saddle point the level sets of $\theta$ exhibits certain self-similar structure. This result was subsequently simpified by Cordoba-Fefferman in $[6]$.

In this paper, we take a different approach first proposed by Deng-Hou-Yu [7] to study the singularity problem of the 3D Euler equations. Following the similar approach for the 2D QG equation, we study the Lagrangian evolution of some localized segments of level sets carrying large $\left|\nabla^{\perp} \theta\right|$. By exploring the incompressibility condition of $\nabla^{\perp} \theta$ and using the local geometric properties of level sets, we obtain estimates for the growth of $\left|\nabla^{\perp} \theta\right|$ by studying the stretching of such level set segments. We find that, when there is one level set segment of length $O\left(\frac{1}{\log \log \|\nabla \theta\|_{L} \infty}\right)$ on which the maximum $\left|\nabla^{\perp} \theta\right|$ is comparable to the global maximum, and along which $\nabla\left(\frac{\nabla^{\perp} \theta}{\left|\nabla^{\perp} \theta\right|}\right)$ is bounded by $O\left(\log \log \|\nabla \theta\|_{L^{\infty}}\right)$, the growth rate of $\left\|\nabla^{\perp} \theta\right\|_{L^{\infty}}$ is bounded by triple exponential. In particular, when the length of the segment and the bound of $\nabla\left(\frac{\nabla^{\perp} \theta}{\left|\nabla^{\perp} \theta\right|}\right)$ are both $O(1)$, we can improve our estimate on the growth of $\left\|\nabla^{\perp} \theta\right\|_{L^{\infty}}$ and bound it by double exponential. The double exponential estimate is sharp according to recent numerical simulations (Ohkitani-Yamada [9], Constantin-Nie-Schorghofer [3], [4]).

We also perform careful numerical experiments to study the local geometric properties of the level sets in a region containing maximum $\left|\nabla^{\perp} \theta\right|$. Our numerical results 
indicate that this region of large $\left|\nabla^{\perp} \theta\right|$ and the region of large $\left|\nabla\left(\frac{\nabla^{\perp} \theta}{\left|\nabla^{\perp} \theta\right|}\right)\right|$ are essentially disjoint. Furthermore, there exists an $O(1)$ level set segment within this region of large $\left|\nabla^{\perp} \theta\right|$ along which $\nabla\left(\frac{\nabla^{\perp} \theta}{\left|\nabla^{\perp} \theta\right|}\right)$ is bounded. Thus our second theorem applies, which implies that $\left\|\nabla^{\perp} \theta(\cdot, t)\right\|_{L^{\infty}}$ is bounded by double exponential in time. In some sense, our theoretical results capture the essential feature of the dynamic growth of the 2D QG equation.

The rest of the paper is organized as follows. In Section 2 we give an overview of the main results. In Section 3 we present numerical results which illustrate the local geometric properties of the level sets in the region containing maximum $\left|\nabla^{\perp} \theta\right|$. Finally, in Section 4 we estimate level set stretching and obtain an estimate for the growth of $\left|\nabla^{\perp} \theta\right|$, and prove the main theorems.

2. Main Results. We present the main results in this section. Denoting $\left\|\nabla^{\perp} \theta(\cdot, t)\right\|_{L^{\infty}}$ by $\Omega(t)$, we consider, at time $t$, a level set segment $L_{t}$ along which the maximum of $\left|\nabla^{\perp} \theta\right|$ (denoted by $\Omega_{L}(t)$ in the following) is comparable to $\Omega(t)$. Denote by $L(t)$ the arc length of $L_{t}, \xi$ the tangential, and $\boldsymbol{n}$ the normal unit vector of $L_{t}$. The direction of $\xi$ and $\boldsymbol{n}$ are determined as follows: $\xi=\frac{\nabla^{\perp} \theta}{\left|\nabla^{\perp} \theta\right|}, \boldsymbol{n}=\frac{\xi \cdot \nabla \xi}{|\xi \cdot \nabla \xi|}$. We define $U_{\xi}(t)=\max _{x, y \in L_{t}}|(u \cdot \xi)(x, t)-(u \cdot \xi)(y, t)|, U_{n}(t)=\max _{x \in L_{t}}|(u \cdot \boldsymbol{n})(x, t)|$, $M(t)=\max _{x \in L_{t}}|\nabla \cdot \xi|$, and $K(t)=\max _{x \in L_{t}} \kappa$ where $\kappa=|\xi \cdot \nabla \xi|$ is the (unsigned) curvature. We should point out that our theorems only requires $L_{t}$ to be a subset of $X\left(L_{t^{\prime}}, t^{\prime}, t\right)$, the flow image of $L_{t^{\prime}}$ at time $t$, for $t^{\prime}<t$. With these notations, we present our main results.

TheOREm 1. Assume that there is a family of level set segments $L_{t}$ and $T_{0} \in$ $\left[0, T_{*}\right)$ such that $X\left(L_{t_{0}}, t_{0}, t\right) \supseteq L_{t}$ for all $T_{0} \leqslant t_{0}<t<T_{*}$. Also assume that $\Omega(t)$ is monotonically increasing and $\Omega_{L}(t) \geqslant c_{0} \Omega(t)$ for some $0<c_{0} \leqslant 1$ for all $t \in\left[T_{0}, T_{*}\right)$. Then the classical solution of the $2 D Q G$ equation can be extended beyond $T_{*}$ as long as the following conditions are satisfied: there exist constants $c_{L}, C_{0}>0$, such that

- $(H 1) . L(t) \geqslant \frac{c_{L}}{\log \log \Omega(t)}$, and

- (H2). $M(t) L(t), \quad K(t) L(t) \leqslant C_{0}$.

Furthermore, for $t \in\left[0, T_{*}\right)$, we have the following triple exponential estimate:

$$
\log \log \log \Omega(t) \leqslant C_{1} t+C_{2}
$$

for some constants $C_{1}, C_{2}>0$ independent of $t$.

REMARK 1. If we further assume that $\Omega(t) \leqslant\left(T_{*}-t\right)^{-B}$ for some $B<+\infty$, we can easily prove non-blowup of the solution with condition (H1) replaced by $L(t) \geqslant$ $c_{L}\left(T_{*}-t\right)^{A}$ for any $A<1$. See Yu [11] for details.

With stronger assumptions on the regularity of $M(t), L(t), K(t)$, we can obtain a sharper growth estimate, which yields double exponential growth of $\Omega(t)$. This growth rate is consistent with the observations in recent numerical simulations (OhkitaniYamada [9], Constantin-Nie-Schorghofer [3], [4]).

THEOREM 2. Assume that all the assumptions and conditions in Theorem 1 hold, except that (H1) is replaced by

- $\left(H 1^{\prime}\right) . L(t) \geqslant c_{L}$.

Then the estimate (6) can be improved to

$$
\log \log \Omega(t) \leqslant C_{1}^{\prime} t+C_{2}^{\prime}
$$


for some constants $C_{1}^{\prime}$ and $C_{2}^{\prime}$ independent of time.

REMARK 2. In Section 3, we will perform numerical experiments to study the local geometric properties of the level sets. In particular, we will show that the conditions (H1') and (H2) are consistent with our numerical results. Furthermore, for certain hyperbolic saddle scenario similar to the one studied by Cordoba in [5] but with additional assumptions, we can prove that the conditions (H1') and (H2) are actually satisfied. This gives a partial theoretical justification of the conditions (H1') and (H2) in certain hyperbolic saddle scenario. The key observation is that the maximum $\left|\nabla^{\perp} \theta\right|$ along any level set stays away from the saddle point. Therefore although $|\nabla \xi|$ is large near the saddle point, it is bounded along level set segments considered in Theorem 2. For details, see Yu [11].

3. Numerical Results. To further understand the dynamics of the $2 \mathrm{D}$ QG system (1)-(3) and to study the local geometric properties of the level sets, we perform careful numerical simulations of the $2 \mathrm{D}$ QG equation. Specifically, we would like to track the dynamic evolution of the following two regions which characterize the geometric regularity of the level sets around the points of maximum $\left|\nabla^{\perp} \theta\right|$ :

1. The region of large $\left|\nabla^{\perp} \theta\right|$, and

2. The region of large $|\nabla \xi|$.

As we will demonstrate from our numerical simulations, these two regions are essentially disjoint. Although they both undergo severe stretching and thining as the flow evolves, their intersection occupies just a small portion of either one of these two regions. This numerical result is quite surprising. It shows that in the very localized region where the maximum of $\left|\nabla^{\perp} \theta\right|$ is attained, the level sets across this region are regular. On the other hand, in the region where $|\nabla \xi|$ is very large, the value of $\left|\nabla^{\perp} \theta\right|$ is relatively small compared with its global maximum. This complementary local geometric regularity of level set filaments seems to be the key in the dynamic depletion of the vortex stretching for the 2D QG equation. Due to this local geometric regularity of level sets, Theorem 2 can be applied to the 2D QG flow, which explains the double exponential growth of $\left|\nabla^{\perp} \theta\right|$ observed in recent numerical simulations.

3.1. Numerical method. Our numerical simulations are performed using the pseudo-spectral method with the $2 / 3$ de-aliasing rule in space, and the 4 th order classical Runge-Kutta in time. We use up to $2048 \times 2048$ space resolution to resolve the rapidly increasing gradient of $\theta$. The size of the time step is determined by the CFL condition. To make the time marching more stable, we use $1 / 6$ of the maximum allowed CFL number. The computation is done on a 4-CPU (Intel(R) Xeon $3.00 \mathrm{GHz}$ ) machine with $2048 \mathrm{~Kb}$ cache and 6G memory. The FFT code is from FFTW 3.1.

We use the same initial condition used by Constantin-Majda-Tabak [2], OhkitaniYamada [9], Constantin-Nie-Schorghofer [3], [4] which contains hyperbolic saddles:

$$
\theta_{0}(x, y)=\sin x \sin y+\cos y .
$$

In Constantin-Nie-Schorghofer [4], other initial conditions were also considered. It was found that the solutions corresponding to these initial conditions grow no faster than the one with the above initial condition, many even exhibit decay of the maximum gradient with time. Therefore we decide to focus on the above initial condition (7) and try to resolve the hyperbolic saddle and the fine structure of the direction field of $\nabla^{\perp} \theta$. 
We plot the maximum $\left|\nabla^{\perp} \theta\right|$ versus time in Figure 1, which is almost identical to Fig. 2 in Ohkitani-Yamada [9]. We also compare the level set contours obtained by our computations with those obtained in Constantin-Nie-Schorghofer [3], [4]. They are essentially indistinguishable.

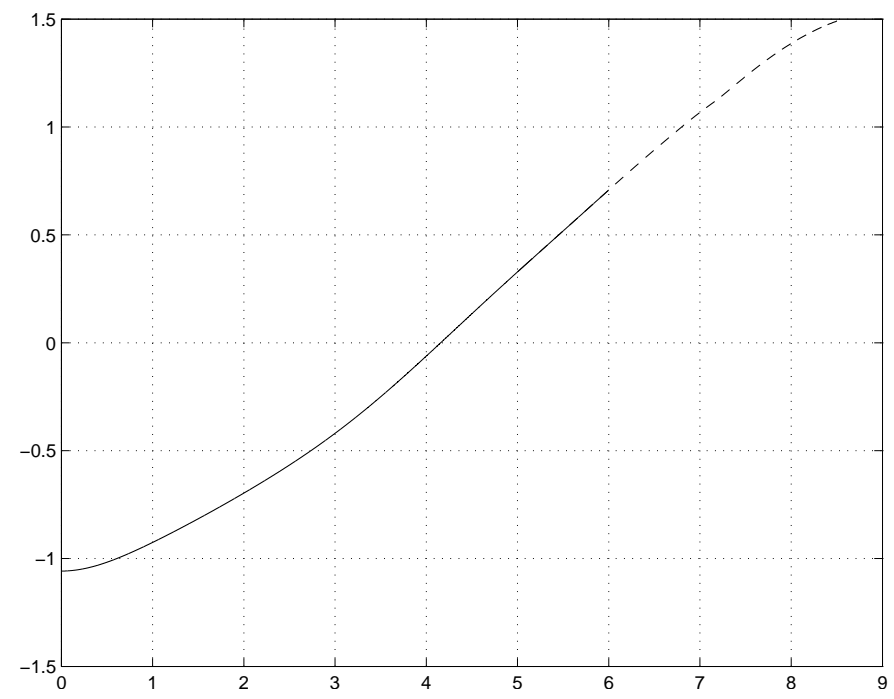

FIG. 1. $\log \log \left|\nabla^{\perp} \theta\right|$ (the base for the logarithm is e) versus time ( $t$ up to about 8.5). The solid curve is obtained using $1024 \times 1024$ resolution $(t=0$ to $t=6)$, and the dashed one is obtained using $2048 \times 2048$ resolution (from $t=5$ on). We see that the two curves are almost identical in the time interval $[5,6]$.

3.2. Dynamics of level set geometries. Next we study the evolution of the two regions mentioned above. We plot, at times $t=5.0,6.0,6.5$, and 7.0 the boundaries of the following two regions:

1. $A_{t} \equiv\left\{(x, y)|| \nabla^{\perp} \theta(x, y, t) \mid \geqslant \frac{1}{2}\left\|\nabla^{\perp} \theta(\cdot, t)\right\|_{L^{\infty}}\right\}$, and

2. $B_{t} \equiv\{(x, y)|| \nabla \xi \mid \geqslant 10\}$.

In Figures $2-6$, we see that $A_{t}$ and $B_{t}$ are essentially disjoint, although some interlacing of the boundaries can be observed. This implies that $|\nabla \xi|$ (consequently $\kappa$ and $\nabla \cdot \xi)$ is bounded in the region where $\left|\nabla^{\perp} \theta\right|$ achieves its maximum. This is an interesting result by itself. It says that the local geometric property of the level sets is regular in the region of maximum stretching. Furthermore, it can be seen from Figures $7-10$ that although $A_{t}$ is severely stretched as time increases, the stretching seems to align with the level set curves. As a result, we can always pick a level set segment of $O(1)$ length, along which the maximum $\left|\nabla^{\perp} \theta\right|$ is comparable to its global maximum. Thus, the conditions of Theorem 2 are satisfied, and we can apply Theorem 2 to conclude that the maximum growth rate of $\left|\nabla^{\perp} \theta\right|$ is bounded by double exponential, which is consistent with recent numerical simulations (Ohkitani-Yamada [9], Constantin-Nie-Schorghofer [3], [4]). 


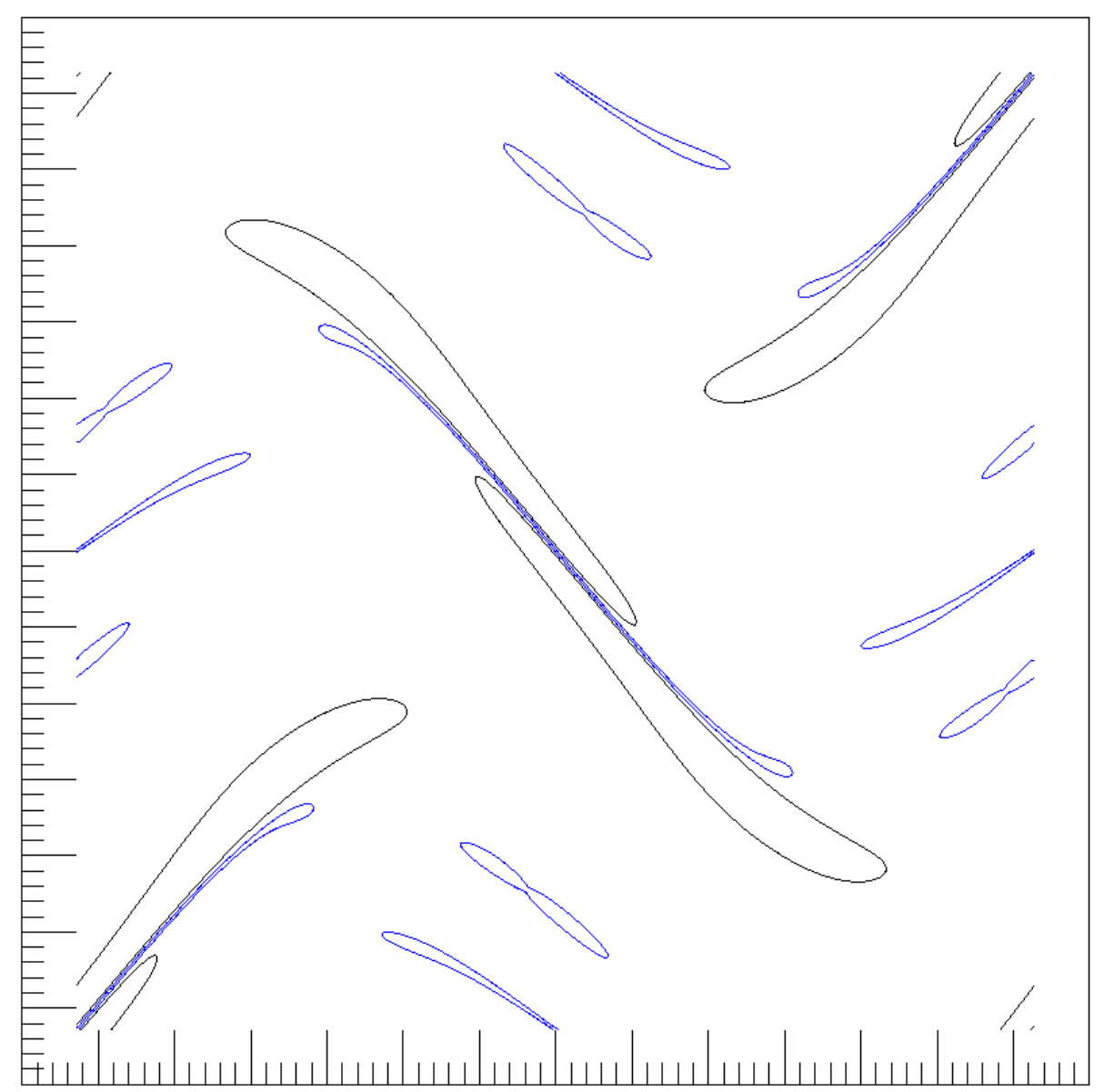

Fig. 2. $A_{t}$ and $B_{t}, t=5.0$. The boundary of $B_{t}$ is plotted in blue and the boundary of $A_{t}$ in black. 


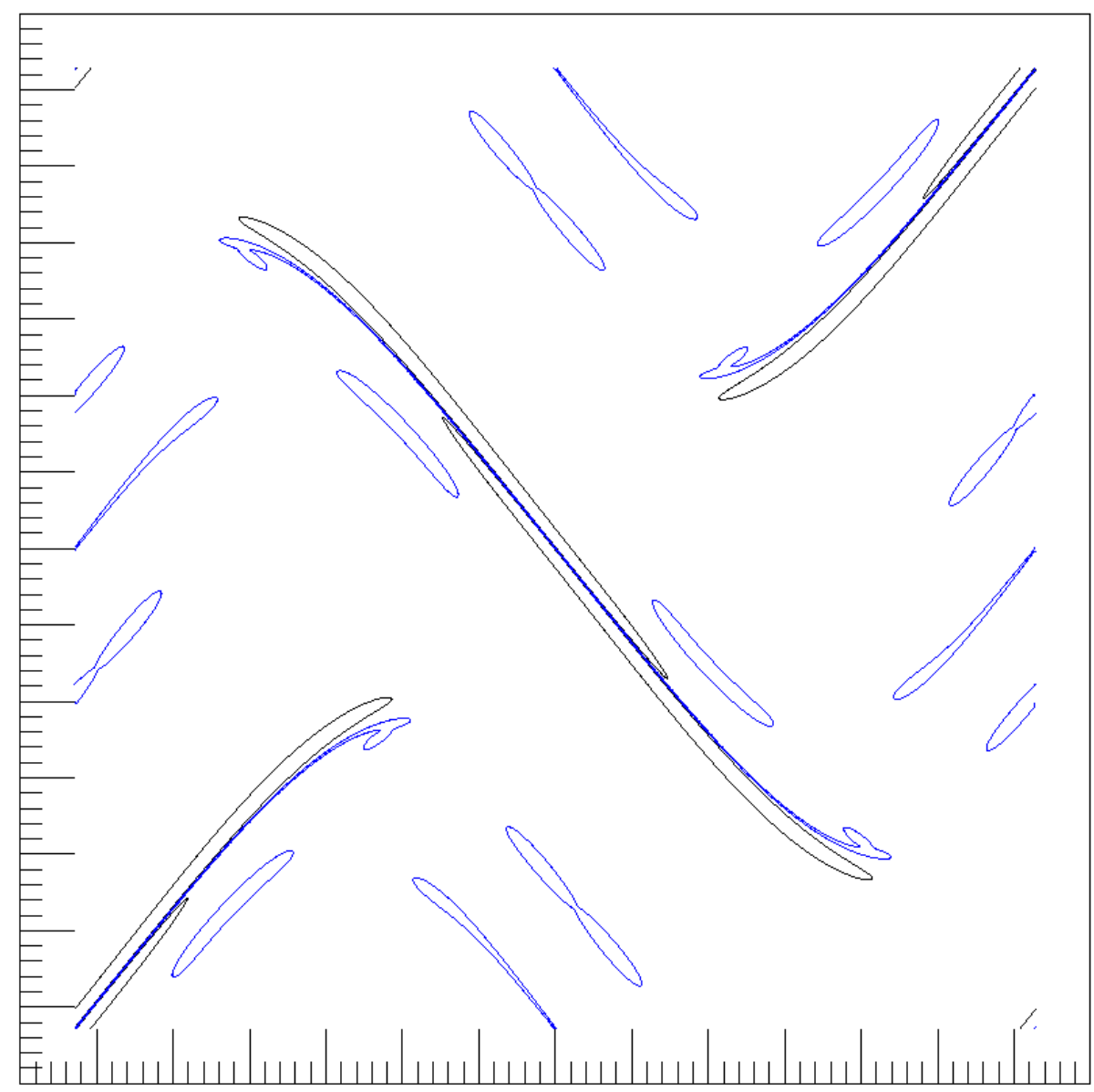

Fig. 3. $A_{t}$ and $B_{t}, t=6.0$. The boundary of $B_{t}$ is plotted in blue and the boundary of $A_{t}$ in black. 
164

J. DENG ET AL.

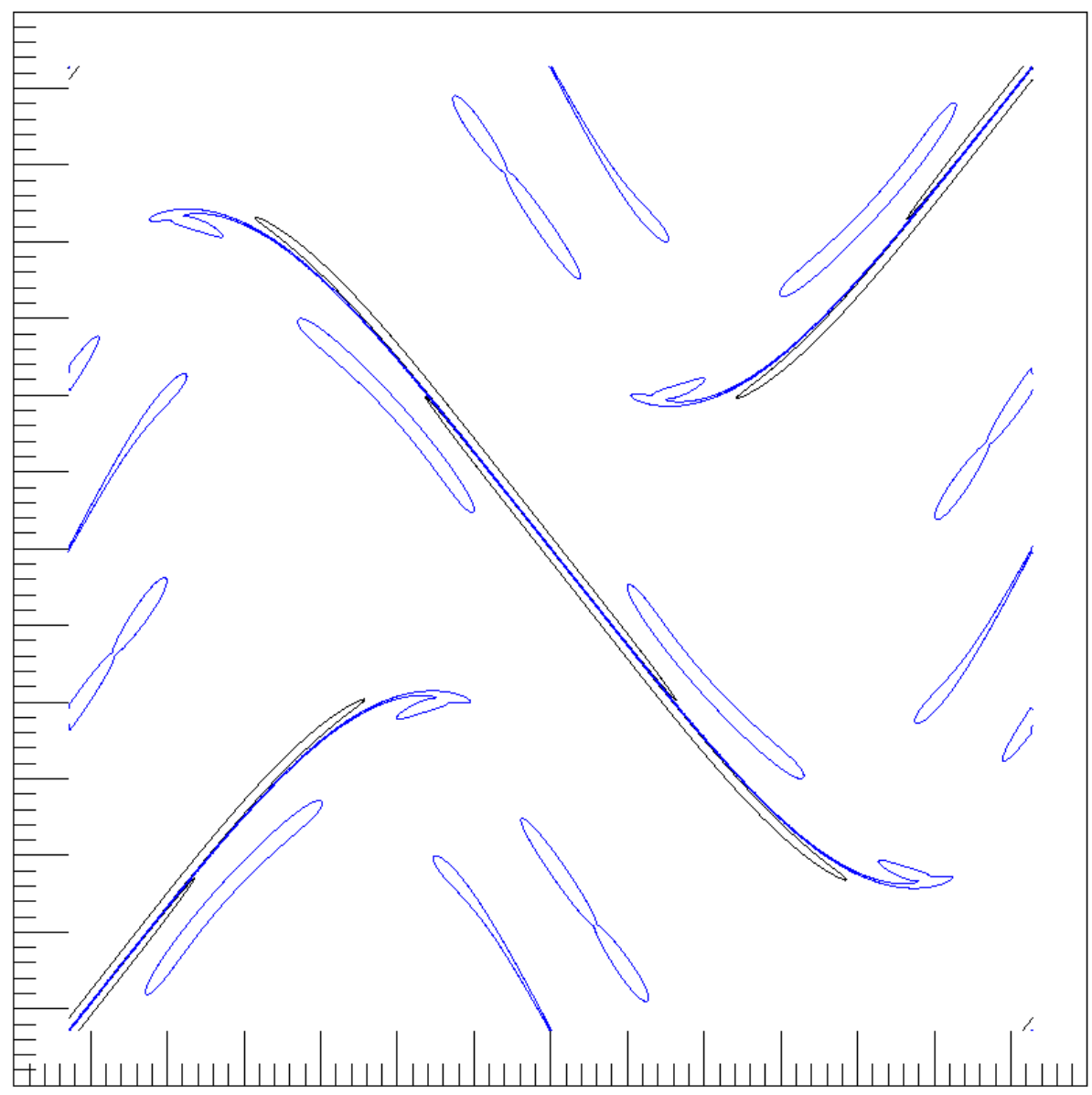

FIG. 4. $A_{t}$ and $B_{t}, t=6.5$. The boundary of $B_{t}$ is plotted in blue and the boundary of $A_{t}$ in black. 


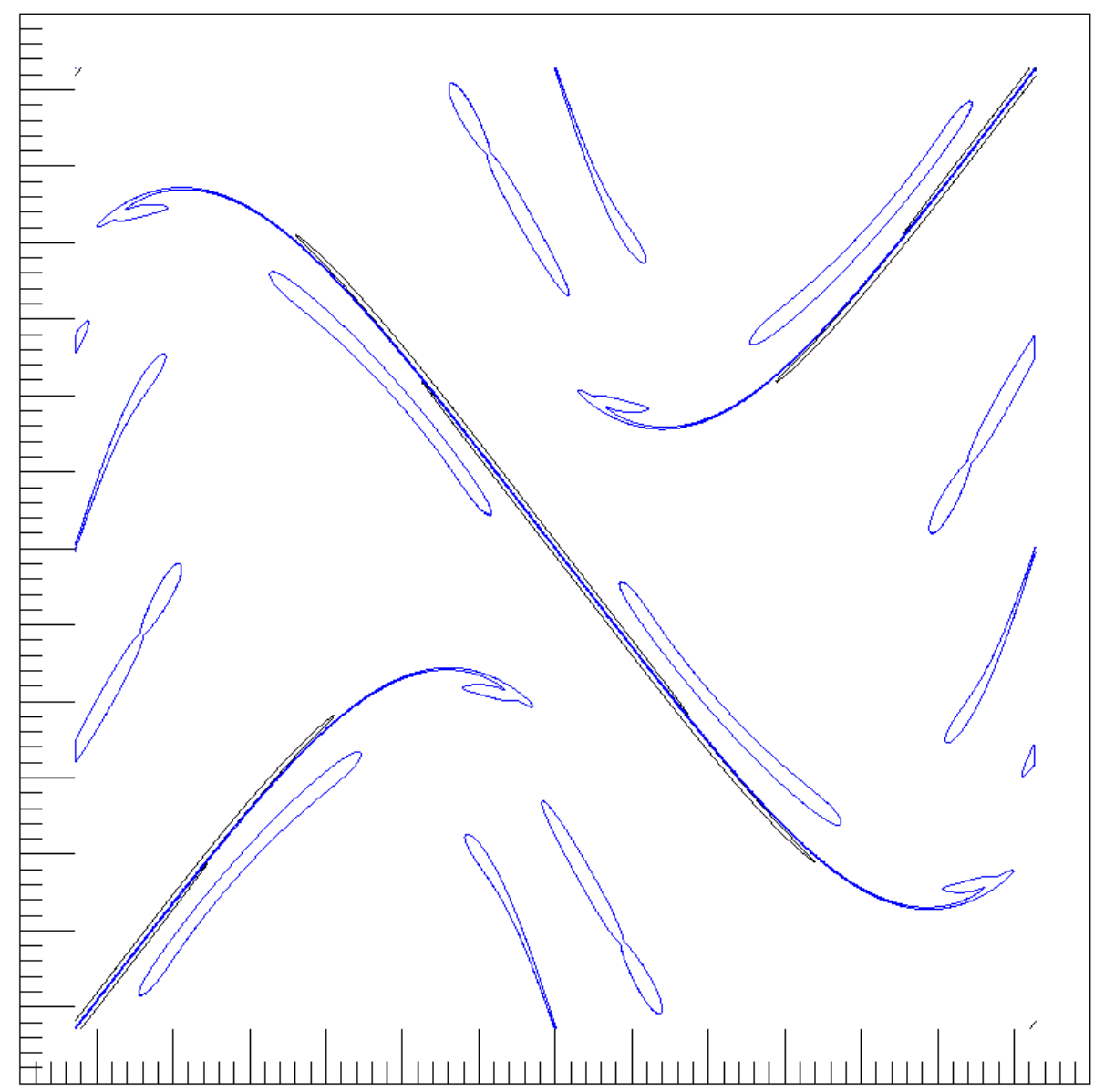

Fig. 5. $A_{t}$ and $B_{t}, t=7.0$. The boundary of $B_{t}$ is plotted in blue and the boundary of $A_{t}$ in black. 


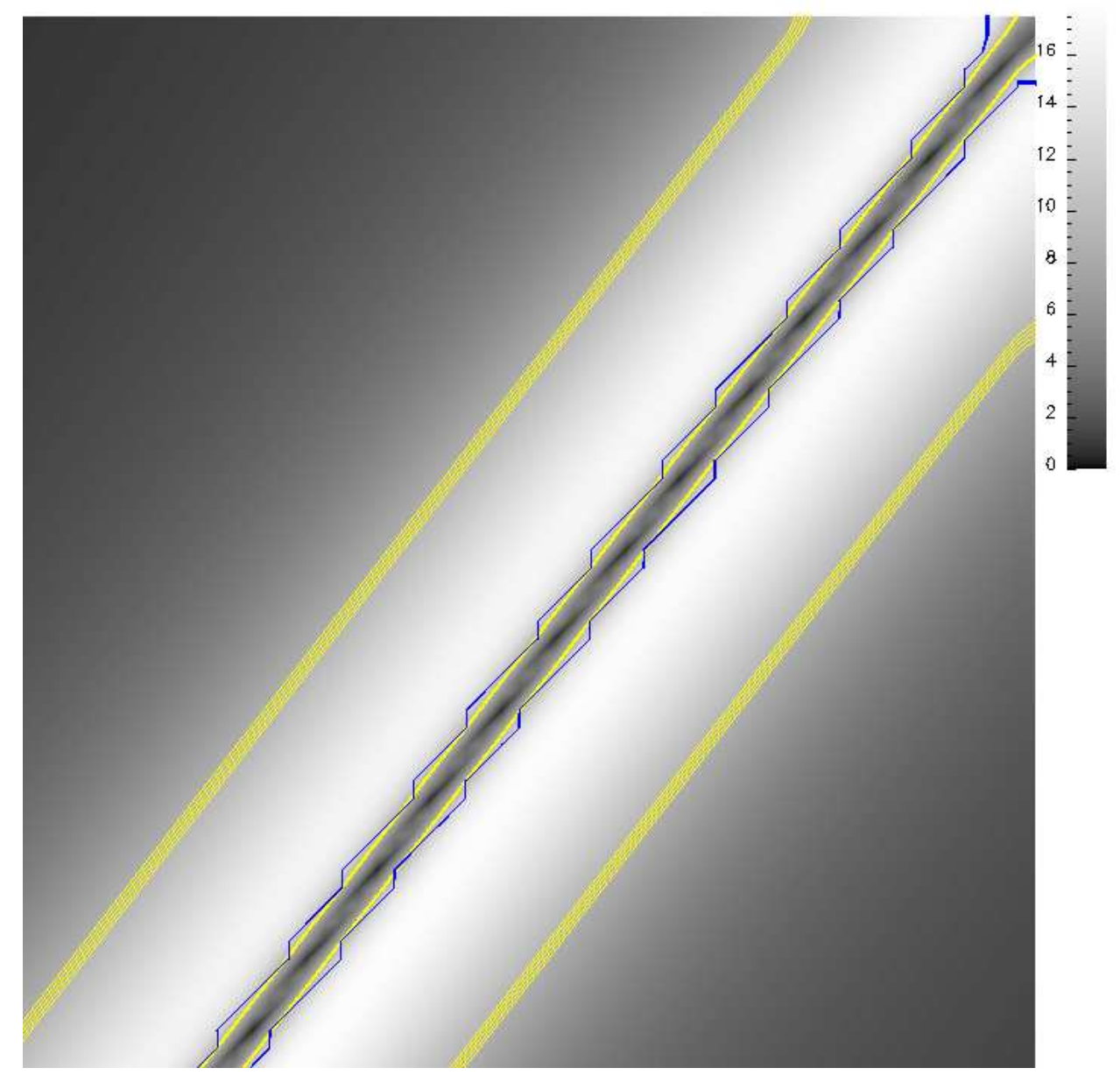

FIG. 6. Zoom in of the upper-right corner of Figure $5(t=7.0)$. Lighter color corresponds to larger $\left|\nabla^{\perp} \theta\right|$. The boundary of $A_{t}$ is plotted in yellow and is thickened by plotting the level sets $\left|\nabla^{\perp} \theta\right|=c\left\|\nabla^{\perp} \theta(\cdot, t)\right\|_{L^{\infty}}$ for $c=0.496,0.498,0.5,0.502,0.504$ for better visibility (This causes the two outer yellow lines to be thicker than the two inner ones, since $\left|\nabla^{\perp} \theta\right|$ changes slower around the two outer lines), and the boundary of $B_{t}$ in blue. We see that basically the two sets $A_{t}$ and $B_{t}$ miss each other. Note that due to periodicity of the data, this figure also reveals what happens in the center part of Figure 5. 


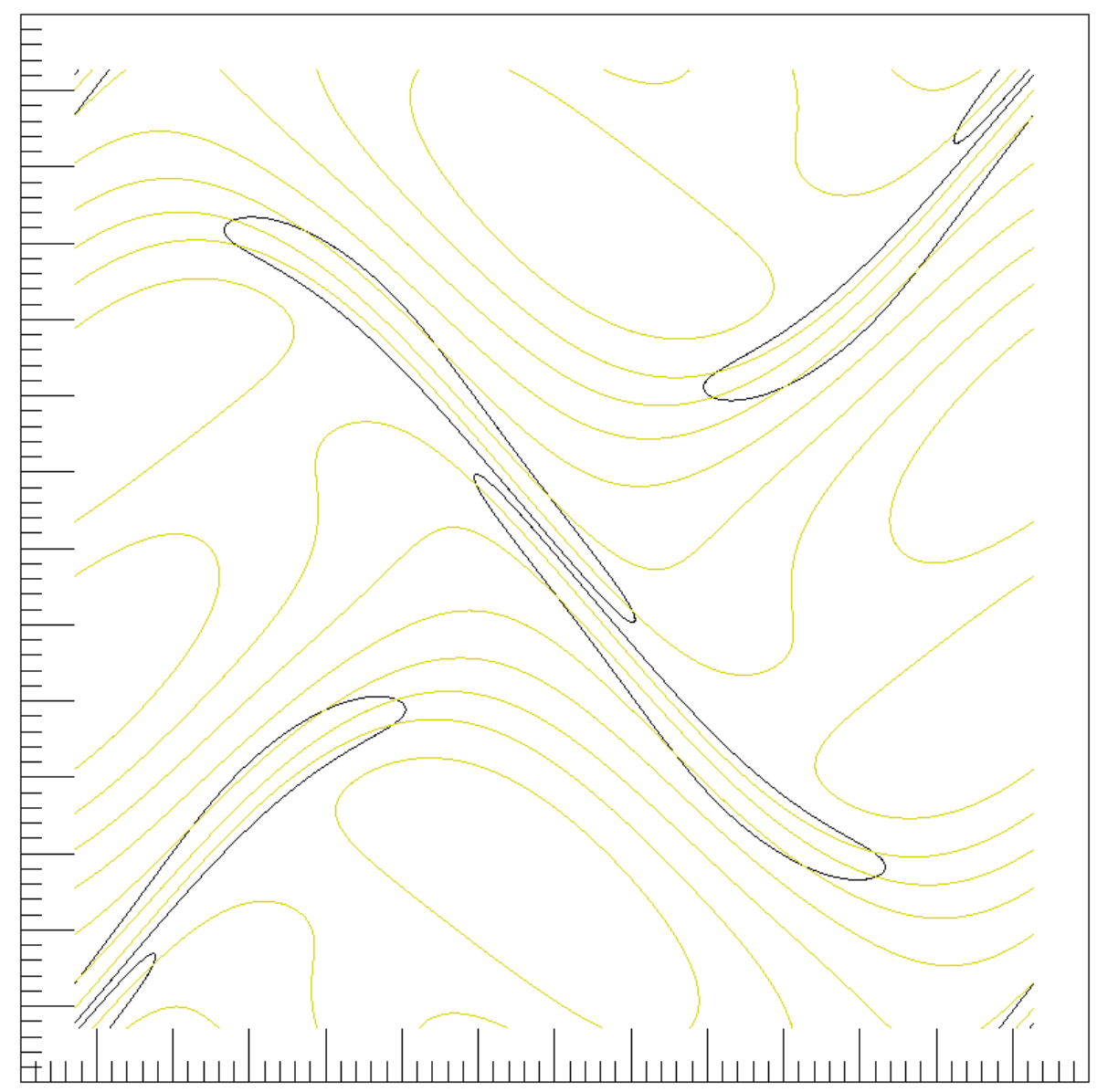

FIG. 7. At (boundary plotted in black) against the level sets of $\theta, t=5.0$. 


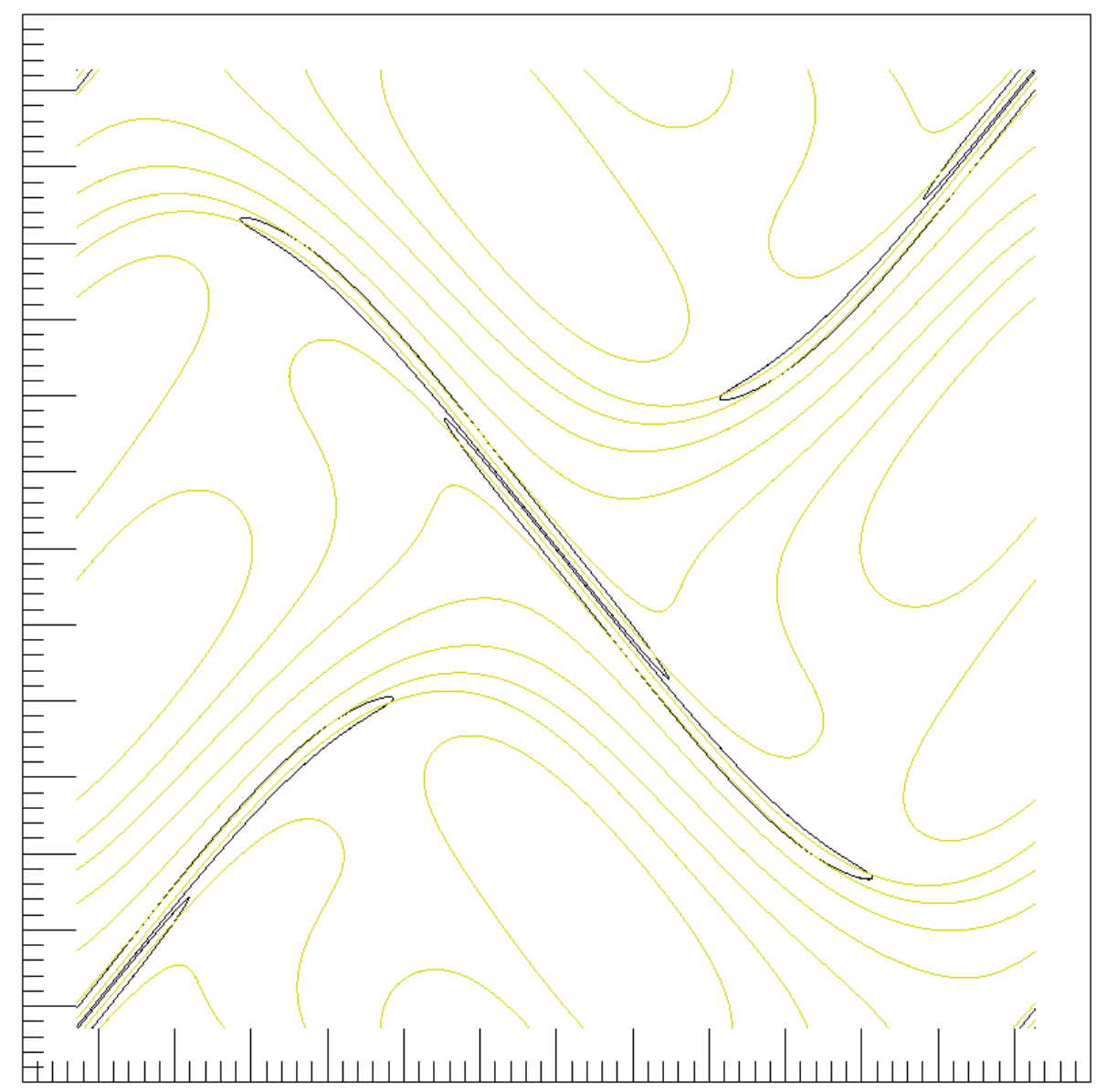

FIG. 8. At (boundary plotted in black) against level sets of $\theta, t=6.0$ 


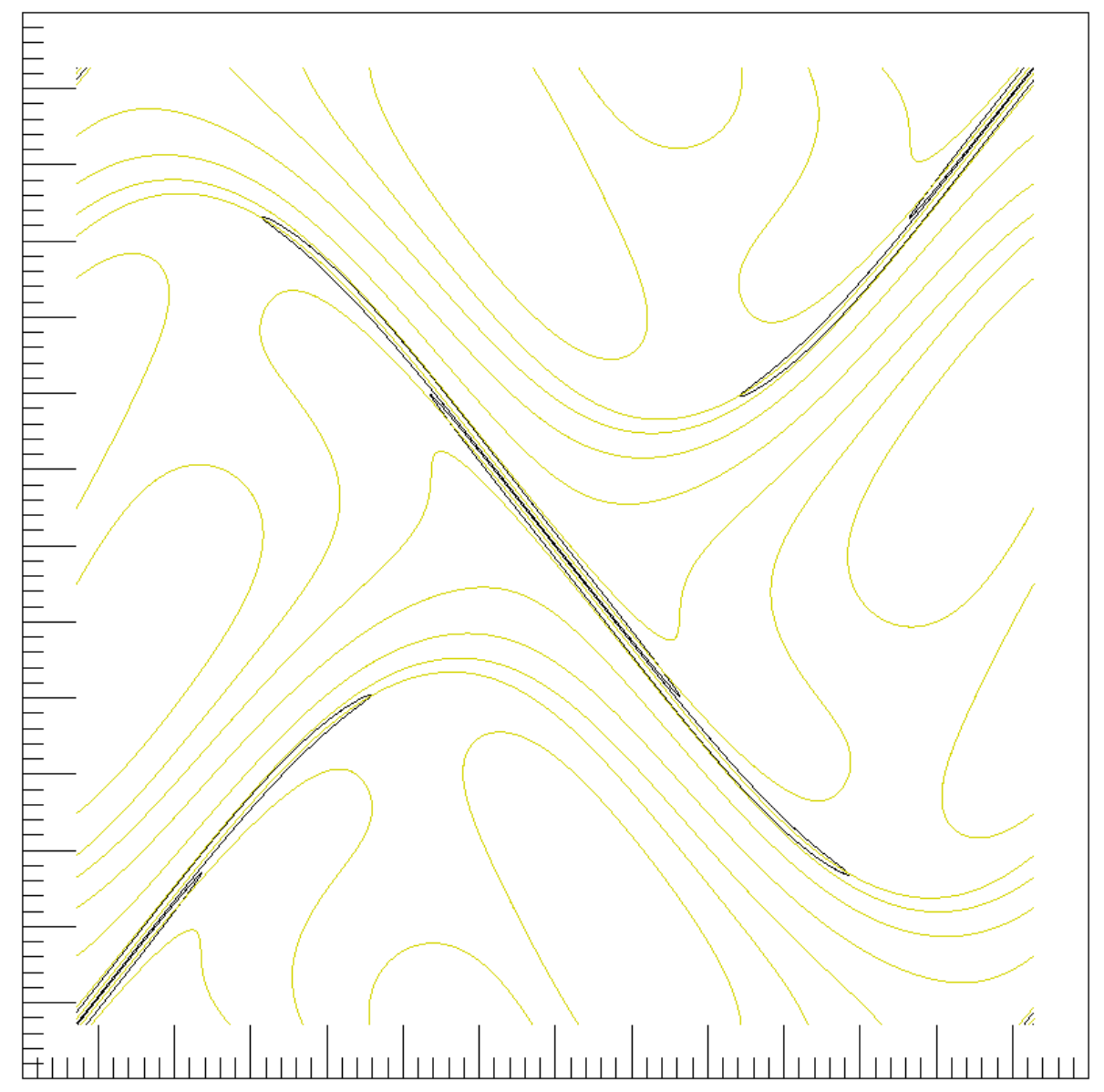

FIG. 9. $A_{t}$ (boundary plotted in black) against level sets of $\theta, t=6.5$ 


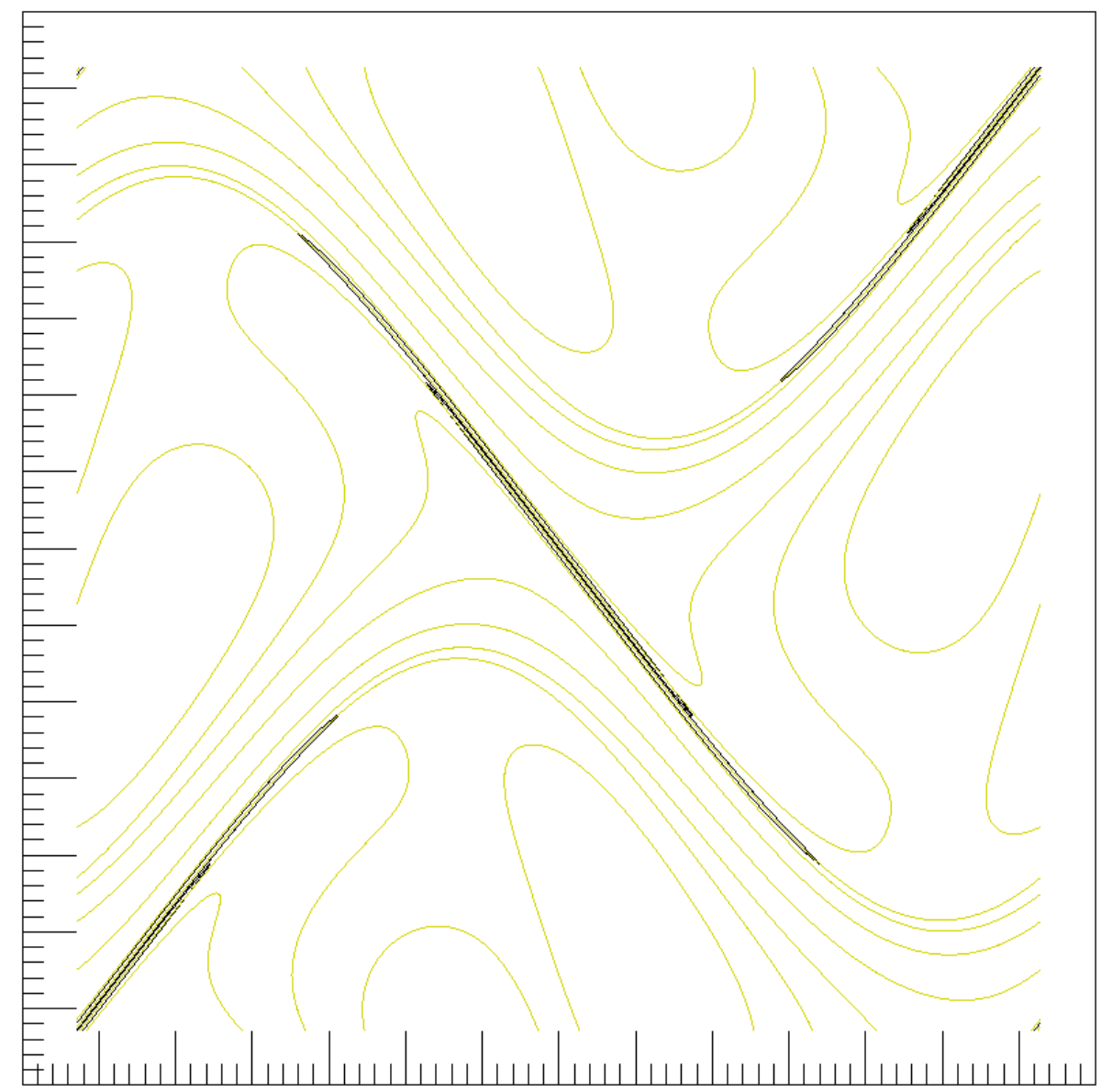

FIG. 10. $A_{t}$ (boundary plotted in black) against level sets of $\theta, t=7.0$

\section{Level Set Dynamics and Proofs of the Theorems.}

4.1. Level set dynamics and key estimate. Before proving the theorems, we need to do some preparations. First we fix notations.

- $C$ or $c$ : generic constants, whose value may change from line to line.

- $\xi$ : the direction of $\nabla^{\perp} \theta \equiv\left(-\frac{\partial \theta}{\partial x_{2}}, \frac{\partial \theta}{\partial x_{1}}\right)^{T}$, that is, $\xi \equiv \frac{\nabla^{\perp} \theta}{\left|\nabla^{\perp} \theta\right|}$ when $\nabla^{\perp} \theta$ does not vanish. In the following we will consider level set segments along which $\left|\nabla^{\perp} \theta\right|$ are comparable to the maximum $\left\|\nabla^{\perp} \theta\right\|_{L^{\infty}}$, therefore we do not need to consider the case when $\xi$ is not well-defined.

- $x, \alpha$ : Cartesian coordinate variables. Thus $x, \alpha \in \mathbb{R}^{2}$.

- $s, \beta$ : arc length variables along the level set under consideration.

- $X(\alpha, \tau, t)$ : the particle trajectory passing $\alpha$ at time $\tau$. In other words, $X(\alpha, \tau, t)$ solves

$$
\begin{aligned}
\frac{\partial X(\alpha, \tau, t)}{\partial t} & =u(X(\alpha, \tau, t), t) \\
X(\alpha, \tau, \tau) & =\alpha .
\end{aligned}
$$


For any set $A \subseteq \mathbb{R}^{2}$, we denote

$$
X(A, \tau, t) \equiv \cup_{\alpha \in A} X(\alpha, \tau, t)
$$

When $\tau=0$, we use the traditional notation $X(\alpha, t) \equiv X(\alpha, 0, t)$.

- $\sim$ : We write $a(t) \sim b(t)$ if there are absolute constants $c, C>0$ such that

$$
c|a(t)| \leqslant|b(t)| \leqslant C|a(t)| .
$$

- $\gtrsim, \lesssim:$ We write $a(t) \gtrsim b(t)$ if there is an absolute constant $c>0$ such that

$$
|a(t)| \geqslant c|b(t)|
$$

$a(t) \lesssim b(t)$ is defined similarly.

In the following subsection, we follow the same line of derivation as in in Deng-Hou-Yu [7]. We give the details of the derivation here to make the presentation self-contained.

4.1.1. Level Set Dynamics. First we derive estimates for the stretching of level sets.

LEMma 1. If $\theta$ solves the $2 D Q G$ equation with initial value $\theta_{0}$, and if furthermore $X(\alpha, t)$ is the flow map, then we have

$$
\nabla^{\perp} \theta(X(\alpha, t), t)=\nabla_{\alpha} X(\alpha, t) \cdot \nabla_{\alpha}^{\perp} \theta_{0}(\alpha) .
$$

where the subscript $\alpha$ denotes partial derivative with respect to $\alpha$.

Proof. We prove by a direct calculation.

$$
\begin{aligned}
\nabla_{\alpha}^{\perp} \theta_{0}(\alpha) & =\nabla_{\alpha}^{\perp} \theta(X(\alpha, t), t) \\
& =\left(\nabla_{\alpha} \theta(X(\alpha, t), t)\right)^{\perp} \\
& =\left(\nabla_{\alpha} X \cdot \nabla \theta\right)^{\perp} \\
& =\left(\nabla_{\alpha} X\right)^{-1} \cdot \nabla^{\perp} \theta(X(\alpha, t), t)
\end{aligned}
$$

where in the last equality we have used the incompressibility property of the flow, i.e. $\operatorname{det} \nabla_{\alpha} X \equiv 1$.

Lemma 2. Consider a point $X\left(\alpha, t_{0}, t\right)$ carried by the flow. Let $s$ be the arc length variable along the level set passing $X\left(\alpha, t_{0}, t\right)$ at time $t$, and let $\beta$ be the arc length variable of the same level set at time $t_{0}$. Then if $\nabla^{\perp} \theta\left(\alpha, t_{0}\right) \neq 0$, we have

$$
\frac{\partial s}{\partial \beta}\left(X\left(\alpha, t_{0}, t\right), t\right)=\frac{\left|\nabla^{\perp} \theta\left(X\left(\alpha, t_{0}, t\right), t\right)\right|}{\left|\nabla_{\alpha}^{\perp} \theta\left(\alpha, t_{0}\right)\right|}
$$

Proof. By Lemma 1 we have

$$
\nabla^{\perp} \theta(X(\alpha, t), t)=\nabla_{\alpha} X(\alpha, t) \cdot \nabla_{\alpha}^{\perp} \theta_{0}(\alpha) .
$$


Therefore

$$
\begin{aligned}
\left|\nabla^{\perp} \theta(X, t)\right| & =\xi(X, t) \cdot \nabla^{\perp} \theta(X, t) \\
& =\xi(X, t) \cdot \nabla_{\alpha} X\left(\alpha, t_{0}, t\right) \cdot \xi\left(\alpha, t_{0}\right)\left|\nabla_{\alpha}^{\perp} \theta\left(\alpha, t_{0}\right)\right| \\
& =\xi(X, t) \cdot\left[\xi\left(\alpha, t_{0}\right) \cdot \nabla_{\alpha} X\left(\alpha, t_{0}, t\right)\right]\left|\nabla_{\alpha}^{\perp} \theta\left(\alpha, t_{0}\right)\right| \\
& =\xi(X, t) \cdot \frac{\partial X}{\partial \beta}\left|\nabla_{\alpha}^{\perp} \theta\left(\alpha, t_{0}\right)\right| \\
& =\xi(X, t) \cdot \frac{\partial X}{\partial s}\left|\nabla_{\alpha}^{\perp} \theta\left(\alpha, t_{0}\right)\right| \frac{\partial s}{\partial \beta} \\
& =[\xi(X, t) \cdot \xi(X, t)]\left|\nabla_{\alpha}^{\perp} \theta\left(\alpha, t_{0}\right)\right| \frac{\partial s}{\partial \beta} \\
& =\left|\nabla_{\alpha}^{\perp} \theta\left(\alpha, t_{0}\right)\right| \frac{\partial s}{\partial \beta}
\end{aligned}
$$

where we have used the fact $\xi(X, t)=\frac{\partial X\left(\alpha, t_{0}, t\right)}{\partial s}$ where $s$ is the arc length variable at time $t$, and in particular, $\xi\left(\alpha, t_{0}\right)=\frac{\partial \alpha}{\partial \beta}$ where $\beta$ is the arc length variable at time $t_{0}$. 口

Now we can write the evolution equation for $\frac{\partial s}{\partial \beta}$ (in the following denoted as $s_{\beta}$ ). In Constantin-Majda-Tabak [2], it is derived that

$$
D_{t}\left|\nabla^{\perp} \theta\right|=(\xi \cdot \nabla u \cdot \xi)\left|\nabla^{\perp} \theta\right| .
$$

Thanks to (9) we immediately have the evolution equation for $s_{\beta}$ :

$$
D_{t}\left(s_{\beta}\right)=(\xi \cdot \nabla u \cdot \xi) s_{\beta}
$$

Now observe

$$
\begin{aligned}
\xi \cdot \nabla u \cdot \xi & =(\xi \cdot \nabla)(u \cdot \xi)-u \cdot(\xi \cdot \nabla) \xi \\
& =(u \cdot \xi)_{s}-\kappa(u \cdot \boldsymbol{n})
\end{aligned}
$$

where we have used $\xi \cdot \nabla=\partial / \partial s$ and the Frenet relation

$$
\frac{\partial \xi}{\partial s}=\kappa \boldsymbol{n}
$$

with $\kappa=|\xi \cdot \nabla \xi|$ being the curvature, and $\boldsymbol{n}$ the unit normal vector of the level set curve. Therefore we have an alternative formulation of the evolution equation of $s_{\beta}$ :

$$
D_{t}\left(s_{\beta}\right)=(u \cdot \xi)_{\beta}-\kappa(u \cdot \boldsymbol{n}) s_{\beta} .
$$

Next we consider a small level set segment at time $t_{0}$, whose two ends are denoted by arc lengths $\beta_{1}<\beta_{2}$. Let $l_{t}$ denote this level set segment at time $t$, and let $l(t)$ denote its length at time $t$. To study its stretching over time, we integrate (12) along $l_{t_{0}}$ to obtain

$$
\begin{aligned}
D_{t}\left[s\left(\beta_{2}, t\right)-s\left(\beta_{1}, t\right)\right]= & (u \cdot \xi)\left(X\left(\beta_{2}, t_{0}, t\right), t\right)-(u \cdot \xi)\left(X\left(\beta_{1}, t_{0}, t\right), t\right) \\
& -\int_{\beta_{1}}^{\beta_{2}}\left[\kappa(u \cdot \boldsymbol{n}) s_{\beta}\right]\left(X\left(\beta, t_{0}, t\right), t\right) \mathrm{d} \beta .
\end{aligned}
$$


Now we further integrate from $t_{0}$ to some later time $t$. We get

$$
\begin{aligned}
s\left(\beta_{2}, t\right)-s\left(\beta_{1}, t\right)= & s\left(\beta_{2}, t_{0}\right)-s\left(\beta_{1}, t_{0}\right) \\
& +\int_{t_{0}}^{t}\left[(u \cdot \xi)\left(X\left(\beta_{2}, t_{0}, \tau\right), \tau\right)-(u \cdot \xi)\left(X\left(\beta_{1}, t_{0}, \tau\right), \tau\right)\right] \mathrm{d} \tau \\
& -\int_{t_{0}}^{t} \int_{\beta_{1}}^{\beta_{2}}\left[\kappa(u \cdot \boldsymbol{n}) s_{\beta}\right]\left(X\left(\beta, t_{0}, \tau\right), \tau\right) \mathrm{d} \beta \mathrm{d} t .
\end{aligned}
$$

Using the notation $l(t) \equiv s\left(\beta_{2}, t\right)-s\left(\beta_{1}, t\right)$, we obtain

$$
l(t) \leqslant l\left(t_{0}\right)+\int_{t_{0}}^{t}\left[u_{\xi}(\tau)+k(\tau) u_{n}(\tau) l(\tau)\right] \mathrm{d} \tau
$$

where $k(\tau)=\max _{l_{t}} \kappa, u_{\xi}(\tau)=\max _{x, y \in l_{\tau}}|(u \cdot \xi)(x, \tau)-(u \cdot \xi)(y, \tau)|$ and $u_{n}(\tau)=$ $\max _{x \in l_{\tau}}|(u \cdot \boldsymbol{n})(x, \tau)|$. Denoting $U(\tau)=\|u(\cdot, \tau)\|_{L^{\infty}\left(\mathbb{R}^{2}\right)}$, we get the following weaker estimate which is enough for our purpose in this paper:

$$
l(t) \leqslant l\left(t_{0}\right)+2 \int_{t_{0}}^{t}[1+k(\tau) l(\tau)] U(\tau) \mathrm{d} \tau .
$$

4.1.2. Estimate of $\left|\nabla^{\perp} \theta\right|$ growth. To apply (14) to the estimate of $\left|\nabla^{\perp} \theta\right|$, we need to relate the stretching of $l_{t}$ to the growth of $\left|\nabla^{\perp} \theta\right|$. This is given by the following two lemmas.

LEMma 3. Let $\xi(x, t)$ be the direction of $\nabla^{\perp} \theta$. Assume at some time $t$, the solution $\theta(x, t)$ is $C^{1}$ in $x$. Then at this time $t$, for any $x$ such that $\nabla \theta \neq 0$, there holds

$$
\frac{\partial\left|\nabla^{\perp} \theta\right|}{\partial s}(x, t)=-\left((\nabla \cdot \xi)\left|\nabla^{\perp} \theta\right|\right)(x, t),
$$

where $s$ is the arc length variable along the level set passing $x$ at time $t$.

Furthermore, if we denote this vortex line by $l$, then for any $y \in l$ such that $\nabla^{\perp} \theta$ does not vanish at any point in the level set segment connecting $x$ and $y$, we have

$$
\left|\nabla^{\perp} \theta\right|(y, t)=\left|\nabla^{\perp} \theta\right|(x, t) e^{\int_{x}^{y}(-\nabla \cdot \xi) \mathrm{d} s},
$$

where the integration is along $l$.

Proof. We compute

$$
\begin{aligned}
0=\nabla \cdot\left(\nabla^{\perp} \theta\right) & =\nabla \cdot\left(\left|\nabla^{\perp} \theta\right| \xi\right) \\
& =(\xi \cdot \nabla)\left|\nabla^{\perp} \theta\right|+(\nabla \cdot \xi)\left|\nabla^{\perp} \theta\right|
\end{aligned}
$$

which immediately gives (15) after noticing $\xi \cdot \nabla=\frac{\partial}{\partial s} \cdot(16)$ is the immediate result of solving (15) along $l$.

LEMMA 4. Let $l_{t}$ be a level set segment carried by the flow, i.e., $l_{t}=X\left(l_{t_{0}}, t_{0}, t\right)$ for some earlier time $t_{0}$. Define

$$
m(t) \equiv \max _{x \in l_{t}}|(\nabla \cdot \xi)(x, t)|
$$


where $\xi=\nabla^{\perp} \theta /\left|\nabla^{\perp} \theta\right|$ is the unit tangent vector. If we further denote $\Omega_{l}(t) \equiv$ $\max _{l_{t}}\left|\nabla^{\perp} \theta\right|$, then the following inequalities hold:

$$
e^{-m(t) l(t)} \frac{\Omega_{l}(t)}{\Omega_{l}\left(t_{0}\right)} \leqslant \frac{l(t)}{l\left(t_{0}\right)} \leqslant e^{m\left(t_{0}\right) l\left(t_{0}\right)} \frac{\Omega_{l}(t)}{\Omega_{l}\left(t_{0}\right)} .
$$

Proof. Recall that $\beta$ is the arc length variable at time $t_{0}$. We have

$$
\begin{aligned}
l(t) & =\int_{\beta_{1}}^{\beta_{2}} s_{\beta} \mathrm{d} \beta \\
& =\int_{\beta_{1}}^{\beta_{2}} \frac{\left|\nabla^{\perp} \theta\left(\alpha, t_{0}, t\right)\right|}{\left|\nabla^{\perp} \theta\left(\alpha, t_{0}\right)\right|} \mathrm{d} \beta \\
& \leqslant \int_{\beta_{1}}^{\beta_{2}} \frac{\Omega_{l}(t)}{e^{-m\left(t_{0}\right) l\left(t_{0}\right) \Omega_{l}\left(t_{0}\right)} \mathrm{d} \beta} \\
& =e^{m\left(t_{0}\right) l\left(t_{0}\right)} \frac{\Omega_{l}(t)}{\Omega_{l}\left(t_{0}\right)} l\left(t_{0}\right),
\end{aligned}
$$

where the inequality is a direct result of (16).

The other inequality is proved similarly. $\mathrm{Q}$

Finally, combining (14) and (17), we obtain the following estimate (after dividing both sides by $\left.l\left(t_{0}\right)\right)$.

$$
\Omega_{l}(t) \leqslant e^{m(t) l(t)} \Omega_{l}\left(t_{0}\right)\left[1+\frac{2}{l\left(t_{0}\right)} \int_{t_{0}}^{t}[1+k(\tau) l(\tau)] U(\tau) \mathrm{d} \tau\right],
$$

where $\Omega_{l}(t) \equiv \max _{l_{t}}\left|\nabla^{\perp} \theta\right|, m(t) \equiv \max _{x \in l_{t}}|(\nabla \cdot \xi)(x, t)|, k(\tau)=\max _{l_{t}} \kappa$, and $U(\tau)=\|u(\cdot, \tau)\|_{L^{\infty}\left(\mathbb{R}^{2}\right)}$. This estimate will play a key role in the proofs of the theorems.

\subsection{Proofs of the Theorems.}

4.2.1. Proof of Theorem 1. . From estimate (18), it is clear that an estimate of $U(\tau)$ is needed. Such an estimate is derived in Cordoba [5]. We summarize his estimate into the following lemma.

Lemma 5. There exists a generic constant $C>0$ such that for $t>0$

$$
\|u(\cdot, t)\|_{L^{\infty}} \leqslant C \log \left\|\nabla^{\perp} \theta(\cdot, t)\right\|_{L^{\infty}}
$$

provided that $\left\|\nabla^{\perp} \theta(\cdot, t)\right\|_{L^{\infty}}>e$.

In our notations, the above estimate is just

$$
U(t) \leqslant C \log \Omega(t) .
$$

Also, we do not need to worry about the condition $\Omega(t)>e$ since we will consider level set segments carrying large $\left|\nabla^{\perp} \theta\right|$.

Now we are ready to prove Theorem 1. First we give a heuristic "proof". By the assumptions of the theorem, we have $m(t) l(t) \leqslant C_{0}$, and $\Omega_{l}(t) \geqslant c_{0} \Omega(t)$. Thus letting $R=e^{C_{0}} / c_{0}$, we have

$$
\Omega(t) \leqslant R \Omega\left(t_{0}\right)\left[1+\frac{C}{l\left(t_{0}\right)} \int_{t_{0}}^{t}[\log \Omega(\tau)+1] \mathrm{d} \tau\right]
$$


where we have used (19). Intuitively, after taking one derivative with respect to $t$, and then setting $t_{0}=t$, we would get

$$
\Omega^{\prime}(t) \leqslant C \Omega(t) \log \Omega(t) \log \log \Omega(t) .
$$

This would give the triple exponential bound. Note that in getting the above differential inequality, we have naively estimated $l\left(t_{0}\right)$ by $\frac{c_{L}}{\log \log \Omega(t)}$. This estimate cannot be derived directly from the assumption $l(t) \geqslant \frac{c_{L}}{\log \log \Omega(t)}$ since in general we only have $l\left(t_{0}\right)<l(t)$. Therefore we need to bound $l\left(t_{0}\right)$ from below using $\Omega(t)$. In the following, we will obtain this lower bound (when $t_{0}$ and $t$ are not far apart) and establish the triple exponential upper bound rigorously.

First we outline the main steps.

- Outline of the main steps. The proof consists of four steps.

1. Divide $\left[T_{0}, T_{*}\right)$ into intervals $\left[t_{k}, t_{k+1}\right)$ such that

$$
\frac{\Omega\left(t_{k+1}\right)}{\Omega\left(t_{k}\right)}=r
$$

for some constant $r>R \equiv e^{C_{0}} / c_{0}$. One of the reasons for doing this partition is to obtain an sharp lower bound estimate for $l\left(t_{k}\right)$ within each time interval $\left[t_{k}, t_{k+1}\right)$ using our relationship between the relative growth of $\Omega(t)$ and the relative growth of arc length stretching between two different times.

2. Use (17) to obtain a lower bound estimate for $l\left(t_{k}\right)$, which in turn gives an upper bound for $\Omega\left(t_{k+1}\right)$ :

$$
\Omega\left(t_{k+1}\right) \leqslant R \Omega\left(t_{k}\right)\left[1+C \frac{\left(1+C_{0}\right) R r}{c_{L}} \log \log \Omega\left(t_{k}\right) \int_{t_{k}}^{t_{k+1}}[\log \Omega(\tau)+1] \mathrm{d} \tau\right] .
$$

3. Use (22) to obtain a local triple exponential estimate for $\Omega\left(t_{k+1}\right)$ :

$$
\begin{aligned}
\log \log \log \Omega\left(t_{k+1}\right) \leqslant & \log \log \log \Omega\left(t_{k+1}\right)+C \frac{R^{2} r\left(1+C_{0}\right)}{c_{L}}\left(t_{k+1}-t_{k}\right) \\
& +\frac{\log R}{\log \Omega\left(t_{k}\right) \log \log \Omega\left(t_{k}\right)} .
\end{aligned}
$$

4. Sum up the estimates for each $\left[t_{k}, t_{k+1}\right)$ to obtain:

$$
\begin{aligned}
\log \log \log \Omega\left(t_{n}\right) \leqslant & \log \log \log \Omega\left(t_{0}\right)+C \frac{R^{2} r\left(1+C_{0}\right)}{c_{L}}\left(t_{n}-t_{0}\right) \\
& +\sum_{i=0}^{n-1} \frac{\log R}{\log \Omega\left(t_{i}\right) \log \log \Omega\left(t_{i}\right)} .
\end{aligned}
$$

It can be shown that the sum in the right hand side can be bounded as follows:

$$
\sum_{i=0}^{n-1} \frac{\log R}{\log \Omega\left(t_{i}\right) \log \log \Omega\left(t_{i}\right)} \leqslant \frac{\log R}{\log r} \log \log \log \Omega\left(t_{n}\right)+C
$$

for some constant $C$. This would give the desired triple exponential estimate for $\Omega\left(t_{n}\right)$ :

$$
\log \log \log \Omega\left(t_{n}\right) \leqslant \frac{\log r}{\log r-\log R}\left[C \frac{R^{2} r\left(1+C_{0}\right)}{c_{L}}\left(t_{n}-t_{0}\right)+C^{\prime}\right] .
$$


Now we carry out the above four steps in detail.

\section{- Partition of the time interval.}

Let $r$ be any constant such that $r>R$ and $t_{0} \in\left[T_{0}, T_{*}\right)$ close enough to $T$ so that $\Omega\left(t_{0}\right)>2 e$ and $\log \log \left(r \Omega\left(t_{0}\right)\right) \leqslant 2 \log \log \Omega\left(t_{0}\right)$. Define $t_{0}<t_{1}<\cdots<$ $t_{k}<\cdots<T_{*}$ by $(21)$, i.e.

$$
\frac{\Omega\left(t_{k+1}\right)}{\Omega\left(t_{k}\right)}=r
$$

If there exists $n \in \mathbb{N}$ such that we cannot find $t_{n+1}$ using (25), or equivalently, such that for any $t \in\left(t_{n}, T^{*}\right)$,

$$
\frac{\Omega(t)}{\Omega\left(t_{n}\right)}<r
$$

then $\Omega(t)$ remains bounded in $\left[0, T_{*}\right]$, and thus no blow-up can occur. Therefore we assume that for all $k \in \mathbb{N}$ we can find $t_{k}$ iteratively such that (25) is satisfied. Since $\lim _{k}>_{\infty} \Omega\left(t_{k}\right)=\infty$ and $T_{*}$ is the smallest time such that $\int_{0}^{T_{*}} \Omega(\tau) \mathrm{d} \tau=\infty$ according to the BKM type criterion (5) derived in Constantin-Majda-Tabak [2], we must have $t_{k} \nearrow T_{*}$.

- Estimate of the lower bound for $l\left(t_{k}\right)$.

We apply (20) to the time interval $\left[t_{k}, t_{k+1}\right]$. for any $t \in\left[t_{k}, t_{k+1}\right]$, choose $l_{t_{k+1}} \subset L_{t_{k+1}}$ so that $\Omega_{l}\left(t_{k+1}\right)=\Omega_{L}\left(t_{k+1}\right)$, and $l\left(t_{k+1}\right)=\frac{c_{L}}{\log \log \Omega\left(t_{k+1}\right)}$, and let $l_{t}$ be such that $l_{t_{k+1}}=X\left(l_{t}, t, t_{k+1}\right)$, i.e., $l_{t}$ is the pullback of $l_{t_{k+1}}$ to time $t \in\left[t_{k}, t_{k+1}\right]$. By the assumptions of Theorem 1 we have $l_{t} \subset L_{t}$ for all $t \in\left[t_{k}, t_{k+1}\right]$. Therefore,

$$
\Omega(t) \leqslant R \Omega\left(t_{k}\right)\left[1+C \frac{1+C_{0}}{l\left(t_{k}\right)} \int_{t_{k}}^{t}[\log \Omega(\tau)+1] \mathrm{d} \tau\right] .
$$

Next we obtain a lower bound for $l\left(t_{k}\right)$. Using (17) we have

$$
\frac{l\left(t_{k+1}\right)}{l\left(t_{k}\right)} \leqslant R \frac{\Omega\left(t_{k+1}\right)}{\Omega\left(t_{k}\right)}=R r
$$

which gives

$$
\frac{1}{l\left(t_{k}\right)} \leqslant \frac{R r}{l\left(t_{k+1}\right)}=\frac{R r \log \log \Omega\left(t_{k+1}\right)}{c_{L}} \leqslant \frac{2 R r \log \log \Omega\left(t_{k}\right)}{c_{L}}
$$

since $\Omega\left(t_{k}\right)>\Omega\left(t_{0}\right)$ is large enough by our choice of $t_{0}$. Thus we obtain the upper bound

$$
\Omega(t) \leqslant R \Omega\left(t_{k}\right)\left[1+C \frac{\left(1+C_{0}\right) R r}{c_{L}} \log \log \Omega\left(t_{k}\right) \int_{t_{k}}^{t}[\log \Omega(\tau)+1] \mathrm{d} \tau\right] .
$$

for all $t \in\left[t_{k}, t_{k+1}\right]$, where $C$ is some absolute constant independent of any parameters.

\section{- Local triple exponential estimate.}

Define $\tilde{\Omega}(t), t \in\left[t_{k}, t_{k+1}\right]$ by

$$
\tilde{\Omega}(t)=R \Omega\left(t_{k}\right)\left[1+C \frac{\left(1+C_{0}\right) R r}{c_{L}} \log \log \Omega\left(t_{k}\right) \int_{t_{k}}^{t_{k+1}}[\log \tilde{\Omega}(\tau)+1] \mathrm{d} \tau\right] .
$$


First we prove $\Omega(t)<\tilde{\Omega}(t)$ for all $t \in\left[t_{k}, t_{k+1}\right]$. When $t=t_{k}$ we have $\tilde{\Omega}\left(t_{k}\right)=R \Omega\left(t_{k}\right)>\Omega\left(t_{k}\right)$. Now suppose that there exists $\delta \in\left(0, t_{k+1}-t_{k}\right]$ so that $\tilde{\Omega}(t)>\Omega(t)$ when $t \in\left[t_{k}, t_{k}+\delta\right)$, and $\tilde{\Omega}\left(t_{k}+\delta\right)=\Omega\left(t_{k}+\delta\right)$. Using (27) and substituting $\tilde{\Omega}\left(t_{k}+\delta\right)=\Omega\left(t_{k}+\delta\right)$ into (28), we obtain

$$
\int_{t_{k}}^{t_{k}+\delta} \log \tilde{\Omega}(\tau) \mathrm{d} \tau \leqslant \int_{t_{k}}^{t_{k}+\delta} \log \Omega(\tau) \mathrm{d} \tau
$$

which contradicts the assumption that $\tilde{\Omega}(t)>\Omega(t)$ when $t \in\left[t_{k}, t_{k}+\delta\right)$ ! Therefore, such $\delta$ cannot exist, which means $\Omega(t)<\tilde{\Omega}(t)$ for all $t \in\left[t_{k}, t_{k+1}\right]$. Next we differentiate (28) with respect to $t$ and get

$$
\tilde{\Omega}^{\prime}(t)=C \frac{R^{2} r\left(1+C_{0}\right)}{c_{L}} \Omega\left(t_{k}\right) \log \log \Omega\left(t_{k}\right)[\log \tilde{\Omega}(t)+1] .
$$

Using $\tilde{\Omega}(t)>\Omega(t)$, we easily obtain

$$
\begin{aligned}
(\log \log \log \tilde{\Omega}(t))^{\prime} & =C \frac{R^{2} r\left(1+C_{0}\right)}{c_{L}} \frac{\Omega\left(t_{k}\right) \log \log \Omega\left(t_{k}\right)[\log \tilde{\Omega}(t)+1]}{\tilde{\Omega}(t) \log \log \tilde{\Omega}(t) \log \tilde{\Omega}(t)} \\
& \leqslant C^{\prime},
\end{aligned}
$$

for some constant $C^{\prime}$. Now integrating (29) over $t$, we obtain a triple exponential growth estimate for $\tilde{\Omega}(t)$.

To obtain the estimate for $\Omega(t)$, notice that $\tilde{\Omega}\left(t_{k}\right)=R \Omega\left(t_{k}\right)$ and $\log \log x$ is a concave function for $x>e^{-1}$, we get

$$
\begin{aligned}
\log \log \log \tilde{\Omega}\left(t_{k}\right) & =\log \log \left(\log R+\log \Omega\left(t_{k}\right)\right) \\
& \leqslant \log \log \log \Omega\left(t_{k}\right)+(\log \log )^{\prime}\left(\log \Omega\left(t_{k}\right)\right) \log R \\
& =\log \log \log \Omega\left(t_{k}\right)+\frac{\log R}{\log \Omega\left(t_{k}\right) \log \log \Omega\left(t_{k}\right)} .
\end{aligned}
$$

Combining with the triple exponential estimate for $\tilde{\Omega}(t)$ and using $\Omega(t)<$ $\tilde{\Omega}(t)$ for $t \in\left[t_{k}, t_{k+1}\right]$, we obtain (24) immediately by taking $t=t_{k+1}$.

- Global estimate.

In the last step we obtain

$$
\begin{aligned}
\log \log \log \Omega\left(t_{k+1}\right) \leqslant & \log \log \log \Omega\left(t_{k}\right)+C \frac{R^{2} r\left(1+C_{0}\right)}{c_{L}}\left(t_{k+1}-t_{k}\right) \\
& +\frac{\log R}{\log \Omega\left(t_{k}\right) \log \log \Omega\left(t_{k}\right)} .
\end{aligned}
$$

Summing over $k$ from 0 to $n-1$, we obtain

$$
\begin{aligned}
\log \log \log \Omega\left(t_{n}\right) \leqslant & \log \log \log \Omega\left(t_{0}\right)+C \frac{R^{2} r\left(1+C_{0}\right)}{c_{L}}\left(t_{n}-t_{0}\right) \\
& \sum_{k=0}^{n-1} \frac{\log R}{\log \Omega\left(t_{k}\right) \log \log \Omega\left(t_{k}\right)} .
\end{aligned}
$$

Now we estimate the sum in the RHS and prove that

$$
\sum_{k=0}^{n-1} \frac{\log R}{\log \Omega\left(t_{k}\right) \log \log \Omega\left(t_{k}\right)} \leqslant \frac{\log R}{\log r} \log \log \log \Omega\left(t_{n}\right)+C
$$


for some constant $C>0, n \geqslant 2$.

Since $\Omega\left(t_{n}\right)=r^{n} \Omega\left(t_{0}\right)$, we have

$$
\begin{aligned}
\sum_{k=0}^{n-1} \frac{\log R}{\log \Omega\left(t_{k}\right) \log \log \Omega\left(t_{k}\right)} & =\sum_{k=0}^{n-1} \frac{\log R}{\log \left(r^{k} \Omega_{0}\right) \log \log \left(r^{k} \Omega_{0}\right)} \\
& =\sum_{k=0}^{n-1} \frac{\log R}{\left(k \log r+\log \Omega_{0}\right) \log \left(k \log r+\log \Omega_{0}\right)} \\
& =\frac{\log R}{\log r} \sum_{k=0}^{n-1} \frac{\log r}{\left(k \log r+\log \Omega_{0}\right) \log \left(k \log r+\log \Omega_{0}\right)}
\end{aligned}
$$

where $\Omega_{0}$ is a shorthand for $\Omega\left(t_{0}\right)$.

Note that the sum

$$
\sum_{k=0}^{n-1} \frac{\log r}{\left(k \log r+\log \Omega_{0}\right) \log \left(k \log r+\log \Omega_{0}\right)}
$$

is in the form of a Riemann sum of the function $(x \log x)^{-1}$. This function is decreasing for $x>e^{-1}$. Therefore the above sum can be bounded by

$$
\begin{aligned}
\int_{\log \Omega_{0}}^{n \log r+\log \Omega_{0}} \frac{1}{x \log x} \mathrm{~d} x & =\log \log \log \left(r^{n} \Omega_{0}\right)-\log \log \log \Omega_{0} \\
& =\log \log \log \Omega\left(t_{n}\right)-\log \log \log \Omega_{0}
\end{aligned}
$$

since $\Omega\left(t_{n}\right)=r^{n} \Omega_{0}$ by our construction. This proves (29).

Now using the fact that $r>R$, we get

$$
\log \log \log \Omega\left(t_{n}\right) \leqslant \frac{\log r}{\log r-\log R}\left[C \frac{R^{2} r\left(1+C_{0}\right)}{c_{L}}\left(t_{n}-t_{0}\right)+C^{\prime}\right],
$$

which implies the triple exponential bound for $\Omega\left(t_{n}\right)$, and consequently no blow-up can occur at time $T_{*}$. This completes the proof of Theorem 1 .

4.2.2. Proof of Theorem 2. The proof is almost the same as that of Theorem 1 . Therefore we will only mention what are different in the four steps of the proof.

1. Partition of the time interval. There is no difference, we still divide $\left[t_{0}, T_{*}\right)$ into sub-intervals such that

$$
\frac{\Omega\left(t_{k+1}\right)}{\Omega\left(t_{k}\right)}=r
$$

for some $r>R$.

2. Estimate of the lower bound of $l\left(t_{k}\right) \cdot(22)$ is replaced by

$$
\Omega\left(t_{k+1}\right) \leqslant R \Omega\left(t_{k}\right)\left[1+C \frac{\left(1+C_{0}\right) R r}{c_{L}} \int_{t_{k}}^{t_{k+1}}[\log \Omega(\tau)+1] \mathrm{d} \tau\right] .
$$

3. Local triple exponential estimate. Define $\tilde{\Omega}$ in a similar way. We obtain

$$
(\log \log \tilde{\Omega}(t))^{\prime} \leqslant C
$$


for some constant $C$. Further noticing that $\tilde{\Omega}\left(t_{k}\right)=R \Omega\left(t_{k}\right)$, and $\log x$ is concave for $x>0$, we have

$$
\begin{aligned}
\log \log \tilde{\Omega}\left(t_{k}\right) & =\log \left(\log R+\log \Omega\left(t_{k}\right)\right. \\
& \leqslant \log \log \Omega\left(t_{k}\right)+\frac{\log R}{\log \Omega\left(t_{k}\right)} .
\end{aligned}
$$

4. Global estimate. From the last step we obtain

$$
\log \log \Omega\left(t_{k+1}\right) \leqslant \log \log \Omega\left(t_{k}\right)+C\left(t_{k+1}-t_{k}\right)+\frac{\log R}{\log \Omega\left(t_{k}\right)}
$$

Therefore

$$
\log \log \Omega\left(t_{n}\right) \leqslant \log \log \Omega\left(t_{0}\right)+C\left(t_{k+1}-t_{k}\right)+\sum_{k=0}^{n-1} \frac{\log R}{\log \Omega\left(t_{k}\right)} .
$$

Recall that $\Omega\left(t_{k}\right)=r^{k} \Omega\left(t_{0}\right)$ by our choice of $t_{k}$. We have

$$
\begin{aligned}
\sum_{k=0}^{n-1} \frac{\log R}{\log \Omega\left(t_{k}\right)} & =\frac{\log R}{\log r} \sum_{k=0}^{n-1} \frac{\log r}{k \log r+\log \Omega_{0}} \\
& \leqslant \frac{\log R}{\log r} \int_{\log \Omega_{0}}^{n \log r+\log \Omega_{0}} \frac{1}{x} \mathrm{~d} x \\
& =\frac{\log R}{\log r}\left[\log \log \Omega\left(t_{n}\right)-\log \log \Omega\left(t_{0}\right)\right]
\end{aligned}
$$

where the inequality is obtained by a similar argument as in the proof of Theorem 1 using the fact that $\frac{1}{x}$ is decreasing for $x>0$. Since $r>R$, we immediately obtain the double exponential estimate

$$
\log \log \Omega\left(t_{n}\right) \leqslant \frac{\log r}{\log r-\log R}\left[C\left(t_{n}-t_{0}\right)+C^{\prime}\right] .
$$

Thus ends the proof.

Acknowledgments. This work was in part supported by NSF under the NSF FRG grant DMS-0353838 and ITR Grant ACI-0204932. We would also like to thank Prof. Norbert Schörghofer for valuable comments.

\section{REFERENCES}

[1] J. Thomas Beale, Tosio Kato, and Andrew J. Majda, Remarks on the breakdown of smooth solutions for the 3-D Euler equations, Comm. Math. Phys., 94:1 (1984), pp 61-66.

[2] Peter Constantin, Andrew J. Majda, and Esteban G. Tabak, Singular front formation in a model for quasigeostrophic flow, Phys. Fluids, 6:1 (1994), pp. 9-11.

[3] Peter Constantin, Qing Nie, And Norbert Schörghofer, Nonsingular surface quasigeostrophic flow, Phys. Lett. A, 241:3 (1998), pp. 168-172.

[4] Peter Constantin, Qing Nie, and Norbert Schörghofer, Front formation in an active scalar equation, Phys. Rev. E (3), 60:3 (1999), pp. 2858-2863.

[5] Diego Cordoba, Nonexistence of simple hyperbolic blow-up for the quasi-geostrophic equation, Ann. of Math. (2), 148:3 (1998), pp. 1135-1152.

[6] Diego Cordoba And Charles Fefferman, Growth of solutions for $Q G$ and $2 D$ Euler equations, J. Amer. Math. Soc., 15:3 (2002), pp. 665-670 (electronic). 
[7] Jian Deng, Thomas Y. Hou, and Xinwei Yu, Geometric properties and nonblowup of 3D incompressible Euler flow, Comm. Partial Differential Equations, 30:1-3 (2005), pp. 225243.

[8] Jian Deng, Thomas Y. Hou, and Xinwei Yu, Improved geometric conditions for non-blowup of the 3D incompressible Euler equation, Comm. PDE, 31 (2006), pp. 293-306.

[9] Koji Ohkitani and Michio Yamada, Inviscid and inviscid-limit behavior of a surface quasigeostrophic flow, Phys. Fluids, 9:4 (1997), pp. 876-882.

[10] Joseph Pedlosky, Geophysical Fluid Dynamics, Springer-Verlag, 1987.

[11] Xinwei Yu, Localized Non-blowup Conditions for 3D Incompressible Euler Flows and Related Equations, PhD thesis, California Institute of Technology, 2005. 